\title{
Acetylcholinesterase Inhibitory Activity of Green Tea Polyphenols
}

\author{
Raghavendra H.L', Prashith Kekuda T.R ${ }^{2}$ and Farhath Khanum ${ }^{3^{*}}$ \\ ${ }^{1}$ College of Medical and Health Sciences, Wollega University, Post Box No: 395, Nekemte, Ethiopia \\ ${ }^{2}$ Post Graduate Department of Studies and Research in Microbiology, Sahyadri Science College \\ (Autonomous), Kuvempu University, Shivamogga-577203, Karnataka, India \\ ${ }^{3}$ Biochemistry and Nano Sciences Division, Defense Food Research Laboratory, Siddarthanagar, \\ Mysore-570011, India
}

\begin{tabular}{ll}
\hline \multicolumn{1}{c}{ Abstract } & Article Information \\
\hline Inhibition of acetylcholinesterase activity is one of the most popular approaches for & Article History: \\
treatment of neurological disorders such as Alzheimer's disease and others. In the & Received :01-11-2014 \\
present study, we evaluated inhibition of acetylcholinesterase activity by different & Revised $: 20-12-2014$ \\
concentrations of green tea (Camellia sinensis L.) extract using acetylthiocholine as & Accepted :26-12-2014 \\
\cline { 2 - 2 } substrate. The green tea extract inhibited AChE activity dose dependently with an & Keywords: \\
$\mathrm{IC}_{50}$ value of $42.05 \mu \mathrm{g} / \mathrm{ml}$. The observed inhibitory activity could be ascribed to the & Green tea polyphenols \\
polyphenolic content of green tea extract. Consumption of green tea might provide & Acetylcholinesterase \\
protection against neurological disorders. & Alzheimer's disease \\
\cline { 2 - 2 } & *Corresponding Author: \\
Copyright@2014 STAR Journal. All Rights Reserved. & Farhath Khanum \\
& E-mail: farhathkhanum@gmail.com \\
\hline
\end{tabular}

\section{INTRODUCTION}

Acetylcholine is a neurotransmitter which plays a key role in memory and cognition. Acetylcholinesterase (AChE) is an enzyme that causes the termination of nerve impulse transmission at the cholinergic synapses by rapid hydrolysis of acetylcholine (ACh). Hence, inhibition of AChE is an important strategy for the treatment of neurological disorders such as Alzheimer's disease, senile dementia, ataxia, myasthenia gravis and Parkinson's disease. Drugs such as tacrine, donepezil, and rivastigmine have been used to treat cognitive dysfunction and memory loss associated with Alzheimer's disease. These drugs have shown to slow down neurodegeneration process. However, these compounds have adverse effects including gastrointestinal disturbances and problems associated with bioavailability. This necessitates an immense interest in searching better AChE inhibitors from natural resources. Ethnopharmacological studies and bioassay-guided isolation have provided a lead in identifying novel and potent AChE inhibitors from plants (Mukherjee et al., 2007; Ohran et al., 2008; Lu et al., 2011).

Tea (Camellia sinensis L., family Theaceae) is one of the most popular beverages consumed all over the world. It is consumed as green, black, or Oolong tea. Among these, significant effects on human health have been observed with the consumption of green tea. Green tea is manufactured by drying fresh tea leaves. Green tea is non-fermented and the beneficial effects of green tea are mainly due to its polyphenols which may account for up to $30 \%$ of dry weight. Catechin is the one of the most important phenolic constituent of green tea. Green tea extract and its components have shown to exhibit activities such as anticancer, hepatoprotective, antimicrobial, antioxidant, neuroprotective and others (Cabrera et al., 2006; Chacko et al., 2010; Jo et al., 2012). In our previous study, we have undertaken an extraction and examination of chemical constituents of green tea for its in vitro antioxidant activity (Raghavendra et al., 2011). The present study focused on the AChE inhibitory activity of green tea extract.

\section{MATERIALS AND METHODS}

Chemicals

Electric eel AChE, Acetylthiocholine iodide and 5-5'thiobis-2-nitrobenzoic acid (DTNB) were purchased from Sigma (USA). Eserine was obtained from Merck (Germany). All other reagents were of analytical grade.

\section{Preparation of Green Tea Extract}

Green tea extract was prepared by following the methodology employed in our previous study (Raghavendra et al., 2011). The green tea extract at different concentrations $(1-100 \mu \mathrm{g} / \mathrm{ml})$ was used to screen AChE inhibitory activity.

\section{AChE Inhibition Assay}

AChE inhibition assay of the green tea extract was carried out according to the method of Orhan et al. (2007) with some modifications. Here, $250 \mu$ l of extract/standard of various concentrations in $200 \mathrm{mM}$ phosphate buffer $(\mathrm{pH}$ 
Raghavendra et alo,

7.7), $80 \mu$ of DTNB (3.96mg of DTNB and $1.5 \mathrm{mg}$ sodium bicarbonate dissolved in $10 \mathrm{ml}$ phosphate buffer $\mathrm{pH} 7.7$ ) and $10 \mu \mathrm{l}$ of enzyme $(2 \mathrm{U} / \mathrm{ml})$ was incubated at $25^{\circ} \mathrm{C}$ for $5 \mathrm{~min}$. After incubation, $15 \mu \mathrm{l}$ of the substrate (acetylthiocholine iodide: $10.85 \mathrm{mg}$ in $5 \mathrm{ml}$ of phosphate buffer) was added and incubated again for $5 \mathrm{~min}$. The color so developed was measured in a microwell plate reader at $412 \mathrm{~nm}$ (Versamax, Molecular Devices, Sunnyvale, USA). The percent inhibition was calculated using the formula:

AChE inhibitory activity $(\%)=(A c-A t / A c) \times 100$, where $A c$ is absorbance of control and $A t$ is absorbance of test. Eserine hemisulphate was used as the standard drug.

\section{Statistical Analysis}

The extract and standard were assayed in triplicate and the result was recorded as Mean inhibition (M.I) \pm Standard deviation (S.D). The $\mathrm{IC}_{50}$ (Inhibitory concentration) values were determined by log-probit analysis.

\section{RESULTS}

The results of the AChE inhibitory activity of green tea extract and standard were depicted in Table 1. Green tea extract showed $66.46 \pm 1.38 \%$ of AChE inhibitory activity at $100 \mu \mathrm{g} / \mathrm{ml}$ concentration and Eserine hemisulphate showed $93.87 \pm 0.96 \%$ of in inhibitory activity at $0.25 \mu \mathrm{g} / \mathrm{m}$. $\mathrm{IC}_{50}$ value of green tea extract and standard was 42.05 and $0.02 \mu \mathrm{g} / \mathrm{ml}$ respectively.

Table 1: AChE inhibitory activity of Green tea extract and standard Eserine hemisulphate

\begin{tabular}{cccc}
\hline Treatment & $\begin{array}{c}\text { Concentration } \\
(\mu \mathrm{g} / \mathrm{ml})\end{array}$ & M.I \pm S.D & $\begin{array}{c}\mathbf{I C}_{50} \\
(\boldsymbol{\mu g} / \mathrm{ml})\end{array}$ \\
\hline Green tea & 1 & $06.41 \pm 1.29$ & \\
extract & 50 & $49.89 \pm 1.43$ & $\mathbf{4 2 . 0 5}$ \\
& 100 & $66.46 \pm 1.38$ & \\
\hline & 0.01 & $28.16 \pm 0.98$ & \\
Eserine & 0.025 & $57.01 \pm 0.77$ & \\
hemisulphate & 0.05 & $73.04 \pm 1.03$ & $\mathbf{0 . 0 2}$ \\
& 0.10 & $87.91 \pm 0.82$ & \\
& 0.25 & $93.87 \pm 0.96$ & \\
\hline
\end{tabular}

Ml: Mean \% inhibition; S.D: Standard Deviation

\section{DISCUSSION}

It is forecasted that $5 \%$ of the global population will be aged 85 years or over by 2034 and it inevitably lead to an increase in age-associated disorders such as Alzheimer's disease (Okello et al., 2012). Alzheimer's disease is one of the neurodegenerative disorders resulted by the loss of cholinergic neuromediators in the brain and enhanced AChE activity. This disease is the most common cause of dementia leading to the loss of intellectual and social abilities severe enough to interfere with daily functioning. The remarkable biochemical change which can be seen in neurodegenerative diseases is the reduction of ACh levels in the hippocampus and cortex of the brain. Therefore, inhibition of AChE is presently the most established approach to treating Alzheimer's disease (Ohran et al., 2008; Okello et al., 2012).
Sci. Technol. Arts Res. J., Oct-Dec 2014, 3(4): 141-142

There is high evidence that green tea exhibits a number of health-promoting effects. It may beneficial potentially to those suffering from neurodegenerative diseases, cardiovascular disease and cancer. The beneficial effects of green tea are mainly attributed to the high polyphenol content in particular catechins (Okello et al., 2012). In the present study, we evaluated AchE inhibitory activity of green tea extract. AChE hydrolyses ACh to give thiocholine and acetate. The reaction between thiocholine and DNBT gives 2-nitro-5-mercaptobenzoate, a yellow compound which is measured at $412 \mathrm{~nm}$. The green tea extract exhibited dose dependent inhibition of AchE activity with an $\mathrm{IC}_{50}$ value of $42.05 \mu \mathrm{g} / \mathrm{ml}$. It has been shown earlier that white and green tea extract and purified tea compounds exhibit AChE inhibitory activity (Okello et al., 2012). The seed and pericarp of tea were shown to possess AChE inhibitory activity (Jo et al., 2012).

\section{CONCLUSION}

The experimental data obtained from the present study showed that green tea extract exhibit potent in vitro AChE inhibition activity. The further in vivo research is undertaken in order to study the exact mechanism of action of green tea polyphenols.

\section{REFERENCES}

Cabrera, C., Artacho, R., Giménez, R. (2006). Beneficial effects of green tea- a review. Journal of the American College of Nutrition 25(2):79-99.

Chacko, S.M., Thambi, P.T., Kuttan, R., Nishigaki, I. (2010). Beneficial effects of green tea: A literature review. Chinese Medicine 5:13.

Jo, Y., Yuk, H., Lee, J., Kim, J., Kim, R., Lee, S. (2012). Antioxidant, tyrosinase inhibitory and acetylcholinesterase inhibitory activities of green tea (Camellia sinensis L.) seed and its pericarp. Food Science and Biotechnology 21(3): 761-768.

Lu, S., Wu, J.W., Liu, H., Zhao, J., Liu, K., Chuang, C., Lin, H., Tsai, W., Ho, Y. (2011). The discovery of potential acetylcholinesterase inhibitors: A combination of pharmacophore modeling, virtual screening, and molecular docking studies. Journal of Biomedical Science 18: 8.

Mukherjee, P.K., Kumar, V., Mal, M., Houghton, P.J. (2007). Acetylcholinesterase inhibitors from plants. Phytomedicine 14: 289-300.

Ohran, I., Kartal, M., Kan, Y., Sener, B. (2008). Activity of essential oils and individual components against acetyl and butyrylcholinesterase. Verlag der Zeitschrift für Naturforschung 63c: 547-553.

Okello, E.J., Leylabi, R., McDougall, G.J. (2012). Inhibition of acetylcholinesterase by green and white tea and their simulated intestinal metabolites. Food and Function 3(6):651-661

Orhan, I., Naz, Q., Kartal, M., Tosun, F., Sener, B., Choudhary, M.I. (2007). In vitro anticholinesterase activity of various alkaloids. Verlag der Zeitschrift für Naturforschung 62c: 684-688.

Raghavendra, H.L., Kumar, P.S.V., Kekuda, P.T.R., Ejeta, E., Molla, B., Anilakumar, K.R., Khanum, F. (2011). HPLC method for chemical composition and in vitro antioxidant activity of Camellia sinensis Linn. Analytical Chemistry Letters 1(5 and 6): 361-369. 